\title{
A Review on Lossy to Lossless Image Coding
}

\author{
Amruta S. Gawande \\ M.Tech Student \\ Dr. Babasaheb Ambedkar Technological University \\ Lonere, Dist. Raigad \\ Maharashtra, India
}

\author{
Sanjay L. Nalbalwar \\ Associate Professor \\ Dr. Babasaheb Ambedkar Technological University \\ Lonere, Dist. Raigad \\ Maharashtra, India
}

\begin{abstract}
With increasing demand for applications in multimedia, mobile communications and computer networks, the field of image coding attracts many researchers. Accomplishment of higher compression ratio while retaining good image quality is needful in the present demanding environment.

Many multimedia applications are demanding for low disk memory requirement, faster and good perceptual quality for images/video. In this paper, authors have reviewed abundant attempts made by researchers to fulfill the requirement of lossy to lossless image coding. One of the best choices for image coding was DCT which is replaced by DWT.

Authors have presented state of art for various methods in lossy to lossless coding domain. With the advancement in research in the fields namely filter banks and lifting based wavelet transforms, image coding with filter banks is currently best suitable method in all aspects.
\end{abstract}

\section{General Terms}

Image coding, algorithm, Block Transform coding,

\section{Keywords}

Filter banks, lifting based wavelet, image compression

\section{INTRODUCTION}

Image compression deals with the problem of reducing the amount of data required to represent an image. Image compression enables the compact representation of an image, thereby reducing the image storage/transmission requirements. With the great advancement in multimedia and internet applications, the demands and requirements of the technologies used, grew and developed. To fulfill these demands and requirements in area of still image compression, many efficient techniques with considerably different features have been formulated for both lossy and lossless compression.

Lossless compression algorithms do not provide high compression ratios. Hence, lossy compression algorithms are preferred for multimedia compression.

There are many lossy compression algorithms developed for image coding such as the classical predictive coding [1], the popular transform coding [2], the wavelet coding [3] and vector quantization [4]. Predictive coding deals with decorrelation of similar neighboring pixels within an image to remove redundancy. Transform coding, an efficient coding scheme is based on employment of inter-pixel correlation which is a core technique recommended by JPEG. Another well-known form of data compression which is adapted for image and audio compression is Wavelet coding. Whereas a technique called vector quantization which is often used in lossy data compression requires preparing of an appropriate codebook to compress data.
One of the most efficient methods of lossy compression of images and other random signals is transform coding.

Image compression began to deal with transform coding from year 1971 with application of discrete Fourier transform for achieving image compression [5]. Usually transform coding uses the Fourier related transforms such as the KL transform [6], HADAMARD transform [7] etc. The representation of the data using smaller number of variables known as Singular value decomposition [8] had been widely used for image compression. In past few decades, Discrete Cosine Transform has been widely used for image coding as it provides optimal performance and can be implemented at a reasonable cost. With the debut of wavelet from recent advances in signal processing tools opened up a new horizon in image coding. Wavelets for image coding applications were used first in the year 1989. The wavelet transform has established beyond consideration to be very effective and has gained popularity over the DCT. Discrete Wavelet Transforms have the skill to solve the blocking effect that sticks in the DCT. From last few years Different wavelets and its variants were used to accomplish better compression. Various transforms have been proved to be reliable for picture coding, including KarhonenLoeve, DCT, lapped orthogonal, lifting based wavelet and filter banks.

This paper focuses on various transform coding methods which combine the advantages of classical methods to improve the respective techniques and accomplish better quality reconstructed image with higher compression ratio. Rest of the paper is organized as follows: section 2 deals with the Performance Measurement, section 3 briefs about traditional techniques of lossy image coding and is devoted to Multirate Filter Banks and Wavelet Approach based Image coding .Discussion \& conclusion of this review is presented in section 4

\section{PERFORMANCE METRICS}

In order to evaluate the performance of image compression systems, a technique to measure compression is needed.

For this purpose, the compression ratio (CR) metric is often employed and is defined as [9]

$$
\mathrm{CR}=\frac{\text { original image size in bits }}{\text { compressed image size in bits }}
$$

Sometimes compression is instead quantified by stating the bit rate (BR) achieved by compression in bpp (bits per pixel). The bit rate after compression and compression ratio are simply related as [9]

$$
\mathrm{BR}=\frac{\text { bits/pixel for originalimage }}{\mathrm{CR}}
$$


In the case of lossy compression, the reconstructed image is only an approximation to the original. The difference between the original and reconstructed signal is referred to as approximation error or distortion. It is most commonly expressed in terms of mean-squared error (MSE) or peaksignal-to-noise ratio (PSNR). These quantities are defined, respectively as stated in [9],

$$
\mathrm{MSE}=\frac{1}{M N} \sum_{i=0}^{M-1} \sum_{j=0}^{N-1}(\hat{x}[i, j]-x[i, j])^{2}
$$

And [9]

$\operatorname{PSNR}=20 \log _{10}\left(\frac{2^{P}-1}{\sqrt{M S E}}\right)$

Where $x$ is the original image with dimensions $M \times N$ having $P$ bpp, and $\hat{x}$ is the reconstructed image. Evidently, smaller MSE and larger PSNR values correspond to lower levels of distortion vary with the amount of compression. In other words, distortion is implicitly a function of rate (i.e., compression ratio). For this reason, plots (or tables) of distortion versus rate are often used to analyze lossy compression performance. Obviously, for any given rate, the lowest possible distortion is desired.

\section{TRADITIONAL LOSSY IMAGE CODING}

\subsection{Karhonen-Loeve Transform (KLT)}

The Karhonen-Loeve transform is a reversible linear transform that utilizes the statistical properties of the vector representation. It optimally decorrelates the input signal. When the transform basis functions are the set of eigenvectors of the autocovariance matrix of the input signal, effects of the quantization error are minimized. These vectors are defined as Karhonen-Loeve transform (KLT) [10]. Though KLT has the minimum bit rate, practically DCT proved to become best choice for still image coding due to ease of hardware computation and very good energy concentration

\subsection{Discrete Cosine Transform (DCT)}

In DCT, the visibility of coding artifacts due to coefficient of quantization may alter due to sensitivity of DCT to phase, depending on the position of an object. As the DCT is a strictly bounded block transform, problems of transform coding at low bit rates, called blocking effect may exist. The blocking effect is a natural consequence of the independent handling of each block. Due to these visible discontinuities in features, the cross block boundaries are observed in images (in the inter frame image coding with motion-compensated frame prediction) though blocking effects are not so disturbing, but are still perceptible [10].

In literature, some methods for the reduction of blocking effects have been discussed [11]-[13]. In [11], two methods were presented: overlapping, and filtering. In the overlapping method [12], redundant information is transmitted for samples in block boundaries, as blocks overlap slightly. At receiver, the reconstructed samples are average of neighboring blocks, in the overlapping areas. The disadvantage of this approach is the total number of samples to be processed is increased, and which further causes of an increase in the bit rate. In filtering method [13], the coding process at the transmitter is maintained same, and at the receiver low boundary pixels are subject to pass through a low-pass filter. However, this method does not increase the bit rate; the signal across block boundaries is blurred. In [13], authors have presented the filtering method that avoids blurring by employing a prefilter at the transmitter [10].

\subsection{Lapped orthogonal Transform (LOT)}

A new family of transforms for blocking signal coding is presented which has similar advantages of the overlapping method cited above, but without an increment in the bit rate. These new transforms collectively referred to as the lapped orthogonal transform, or LOT [10],

In traditional block-transform processing, such as in image and audio coding, the signal is partitioned into blocks of $\mathrm{M}$ samples, and each block is made to process independently, it is well known that under an orthogonal transformation ,signal energy is preserved,[14] assuming stationary signals, i.e.,

$$
\mathrm{M} \sigma_{\mathrm{x}}^{2}=\sum_{i=0}^{M-1} \sigma_{i}^{2}
$$

Where $\sigma_{i}^{2}$ the variance of output is transform coefficient and $\sigma_{x}^{2}$ is the variance of the input samples.

For lapped transforms [15], the basis vectors can have length $\mathrm{L}$, such that $\mathrm{L}>\mathrm{M}$. Traditional block boundaries are extended to the length of basis vector which results into the non-square transform matrix and the equations those are valid for block transforms couldn't applied to a Lapped Transform, Concentrating efforts on orthogonal Lapped Transform. Consider $\mathrm{L}=\mathrm{NM}$, where $\mathrm{N}$ is the overlap factor. Note that $\mathrm{N}$, $\mathrm{M}$, and hence $\mathrm{L}$ are all integers. In the case of block transforms, transform matrix is defined as matrix containing the orthonormal basis vectors as its rows. A lapped transform matrix $\mathrm{P}$ of dimensions $\mathrm{M} \times \mathrm{L}$ can be divided into

Square $\mathrm{M} \times \mathrm{M}$ sub matrices $\mathrm{P}_{\mathrm{i}}(\mathrm{i}=0,1 \ldots \mathrm{N}-1)$ as

$$
p=\left[\begin{array}{llll}
p_{0} & p_{1} & \cdots & p_{N-1}
\end{array}\right]
$$

The orthogonality property does not hold because $\mathrm{P}$ is no longer a square matrix and it is replaced by the perfect reconstruction (PR) property[15], defined by

$$
\sum_{i=0}^{N-1-l} p_{i}+p_{i+l}^{T}=\sum_{i=0}^{N-1-l} p_{i+l}^{T} p_{i}=\delta(l) I_{M}
$$

for $l=0,1, \ldots, \mathrm{N}-1$, where $\delta(l)$ is the Kronecker delta, i.e., $\delta(0)=1$ and $\delta(l)=0$ for $l \neq 0$ here,(7) states the PR conditions and orthogonality of the transform operating over the entire signal, Showing that energy is preserved, such that (5) is valid.

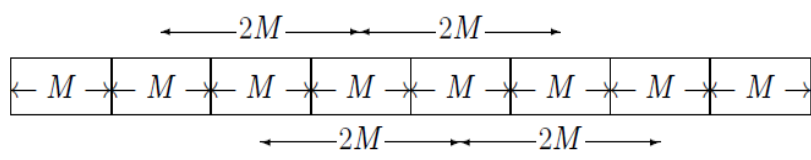

Fig. 1 the signal samples are divided into blocks of $M$ samples. The lapped transform uses neighboring blocks samples, as in this example for $\mathrm{N}=2$, i.e. $\mathrm{L}=2 \mathrm{M}$, yielding an overlap of $(L-M) / 2=M / 2$ samples on either side of a block [10]. 


\subsection{Generalized Linear-Phase LOT (GenLOT)}

The LOT is a popular Lapped transform with $\mathrm{N}=2$ whose basis functions are symmetric [16], its transform matrix, representing its fast algorithm, is given by

$$
\boldsymbol{P}_{L O T}=\left[\begin{array}{cc}
U_{1} & 0 \\
0 & V_{1}
\end{array}\right]\left[\begin{array}{cc}
D_{e}-D_{o} & \left(D_{e}-D_{o}\right) J_{M / 2} \\
D_{e}-D_{o} & -\left(D_{e}-D_{o}\right) J_{M / 2}
\end{array}\right] \text { (8) }
$$

Where $D_{e}$ is the $M / 2 \times M$ matrix with the even-symmetric basis functions of the DCT and $D_{0}$ is the matrix with the odd-symmetric ones. Examining the symmetries of $D^{H}$, it is easy to see that $D_{e}$ and $D_{0}$ have the entries $d_{i j}^{H}$ for i even and odd, respectively. $U_{1}$ and $V_{1}$ are $M / 2 \times M / 2$ orthogonal matrices. $U_{1}=I_{M / 2}$, and approximates $V_{1}$ by $M / 2-1$ plane rotations [16]

The GenLOT is defined as a Linear phase paraunitary Filter banks obeying

$$
E(z)=K_{N-1}(z) K_{N-2}(z) \ldots . . K_{1}(z) E_{0}
$$

Where $E_{0}$ is chosen to be the DCT matrix [17], which is denoted as $D^{H}$. The output of the DCT is then separated into the groups of even and odd coefficients. The GenLOT with $\mathrm{N}$ - 1 stages after the DCT has basis functions (filters) with length $\mathrm{L}=\mathrm{NM}$ and has its Polyphase Transfer Matrix defined as

$$
E(z)=K_{N-1}(z) K_{N-2}(z) \ldots . . K_{1}(z) D^{H}
$$

\subsection{Discrete Wavelet Transform (DWT)}

The main advantage of wavelet transforms over other more traditional decomposition methods (like the DCT) is that the basis functions associated with a wavelet decomposition typically have both long and short support. The basis functions with long support are effective for representing slow variations in an image while the basis functions with short support can efficiently represent sharp transitions (i.e., edges)[19].

This makes wavelets ideal for representing signals having mostly low-frequency content mixed with a relatively small number of sharp transitions. The wavelet transform decomposes a signal into frequency bands that are equally spaced on a logarithmic scale. The low-frequency bands have small bandwidths, while the high-frequency bands have large bandwidths. This logarithmic behavior of wavelet transforms can also be advantageous. Since human visual perception behaves logarithmically in many respects, the use of wavelet decompositions can sometimes make it easier to exploit
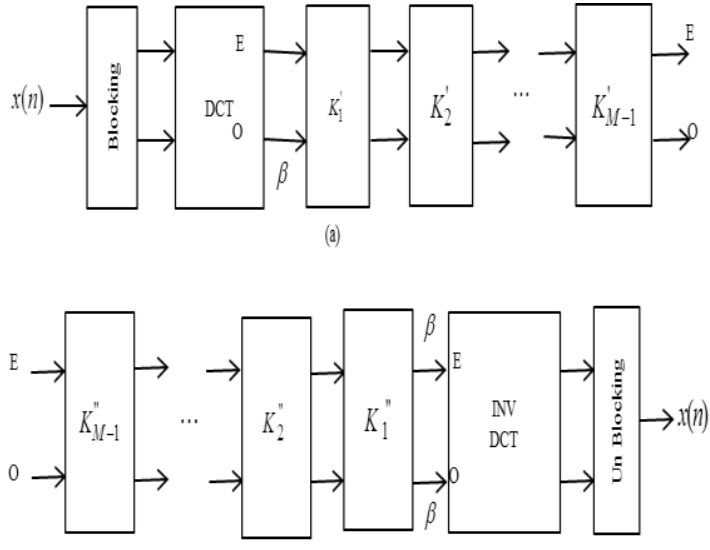

(b)

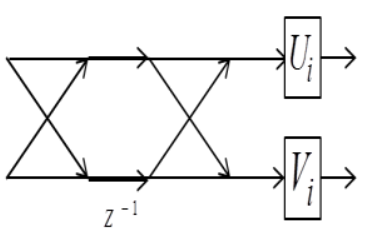

(c)

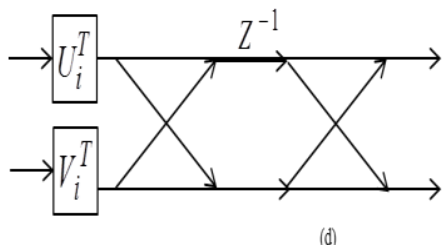

(d)
Fig.2 Flow-graph for implementation of GenLOT Each branch carries $M / 2$ samples and $E$ and $O$ stand for even and odd transform coefficients, respectively, of output (analysis) and input (synthesis) for both DCT and GenLOT. Even and odd coefficients also correspond to symmetric and anti-symmetric basis function (which are the filters impulse response), respectively. $\beta$ is scaling factor incorporating all (a) Analysis (b) Synthesis(c) Detail of the Analysis stages $K_{i}^{\prime}$ for $\mathrm{M}=8$ (d) Details of synthesis

stages $K_{i}^{\prime \prime}$ for $\mathrm{M}=\mathbf{8}[18]$

characteristics of the human visual system in order to obtain improved subjective lossy compression results. Although wavelet transforms with many different characteristics are possible, orthogonal transforms with symmetric finitelysupported basis functions are ideally most desirable for image compression.

In Wavelet based coding, advantage of overlapping basis function and better energy compaction property of wavelet transform is utilized to provide subsequent advancement in picture quality at low bit rate. Because of built-in multi resolution nature, wavelet based coder facilitates progressive transmission of images with grant for variable bit rate [20].

In [21], wavelets which are strictly translates and dilates of each other but still maintain all the powerful properties of first generation wavelets is presented, referring to these wavelet as second generation wavelet known as lifting scheme. The lifting scheme is a simple yet powerful tool to construct second generation wavelets. The lifting scheme is an alternative description of the discrete wavelet transform as wel as an alternative way to build wavelets. Lifting provides several advantages including:

- In-place calculation of the wavelet coefficients

- Inverse wavelet transform is easily obtained

- Ability to perform integer-to-integer wavelet transforms 
- Extension to domains, which are not shift-invariant.

- Extension to irregularly-sampled data

Lifting scheme directs to a faster, in-place calculation of the wavelet transform [22]

\section{FILTER BANKS FOR IMAGE CODING}

Filter banks are used to separate the input signal into multiple components, each component carrying a single frequency Subband of the original signal [23], [24]. It is also desirable to design the filter bank such that these subbands can be recombined to recover the original signal.

Ideal filters, inherently, are not feasible and the issue was first addressed using two-channel linear-phase filter banks and a design called quadrature mirror filter bank(QMF) was introduced to cancel aliasing resulting from the decimation and interpolation processes [23], [25]. The so called Johnston's filters are a family of QMF designed for this approach [26]

The QMF solutions do not allow perfect reconstruction (PR) of the signal and later Smith and Barnwell [27] developed the conjugate quadrature filter bank (CQF) in a formulation which does not use linear-phase filters but allows PR of the signal. Both QMF and CQF solutions have a two-channel filter bank which can be hierarchically associated in a binary-tree path in order to create filter banks with more than two channels.

A uniform filter bank is the one where all, let us say, $\mathrm{M}$ filters have bandpass width of $\pi / \mathrm{M}$, thus signals of all Subband are decimated and interpolated by a factor of M [23],[24]. Fig. 3 shows an M-channel critically decimated uniform filter bank. In this figure, $M$ is the number of filters (or number of channels or Subband), $\mathrm{x}(\mathrm{n})$ is the input signal, and $\hat{x}$ (n) is the recovered signal after synthesis. The Subband signals are represented by $y_{i}(m) \quad(0 \leq \mathrm{i} \leq \mathrm{M}-1)$, and the filters with impulse responses $f_{i}(m)$ and $g_{i}(m) \quad(0 \leq \mathrm{i} \leq \mathrm{M}-1)$ correspond to analysis and synthesis sections, respectively.

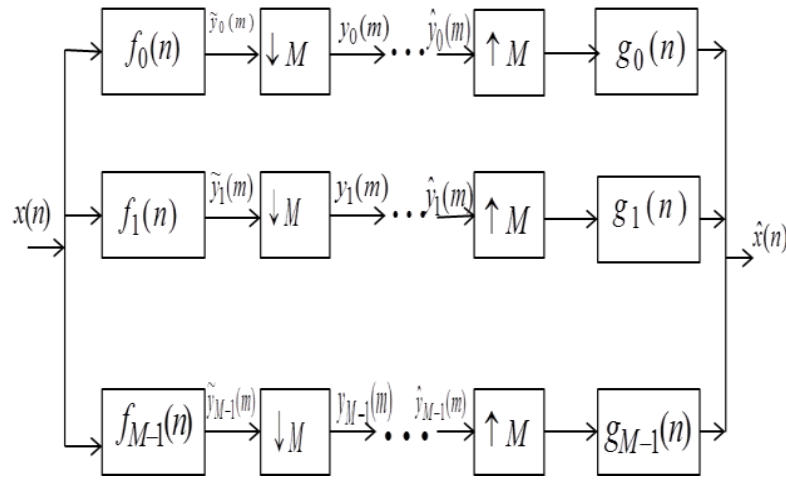

Fig 3: Critically decimated uniform filter bank. Analysis (left) and synthesis (right) section [24].

Filter banks can also be classified into paraunitary or biorthogonal [24]. In paraunitary FIR filter banks, each $f_{i}(m)$ has a one-to-one correspondence to $g_{i}(m)$ [15], [25], [28], while in bi-orthogonal filter banks the set $f_{i}(m)$ is found from the entire set of $g_{i}(m)$ or vice versa [24], [28]. This is similar to the relation between orthogonal and non-orthogonal matrices, and in fact, orthogonal block transforms are a special case of paraunitary filter banks, while non-orthogonal ones belong to the class of bi-orthogonal filter banks.

In numerous applications, especially image processing, it is crucial that all analysis and synthesis filters have linear phase. Such system is called a linear phase filter bank (LPFB). Besides the elimination of the phase distortion which is often disastrous in many image processing applications [30], LP filters preserve the locality of the edges, the key to success of hierarchy image coding algorithms [28], [29], [31], [32].

The latter Polyphase representation in Figure 4(b) proves to be very useful, both theoretically and practically, in filter bank design and application.
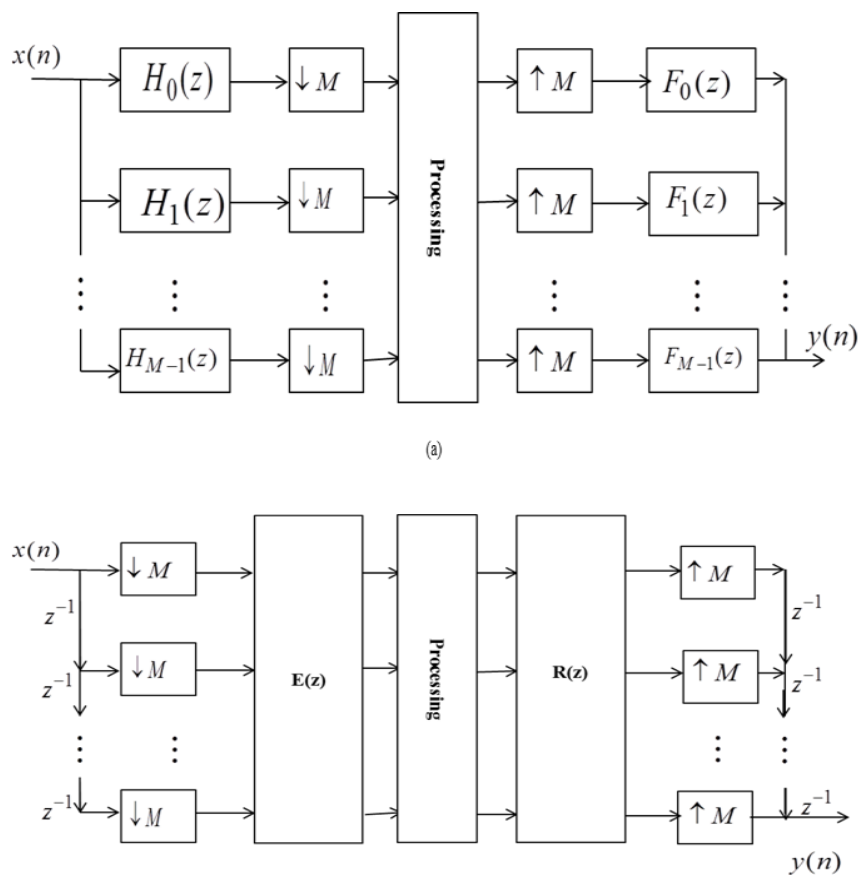

Fig 4: M-channel filter bank a) regular structure b) Polyphase structure

Not only does it allow the processing of signals at lower rates, but it also simplifies filter bank theory dramatically.

\subsection{Paraunitary filter banks (PUFBs)}

The design and implementation of orthogonal systems are based on multi-input and multi-output systems, of which the Polyphase transfer matrices are paraunitary. A Polyphase transfer function matrix $\mathrm{E}(\mathrm{z})$ is paraunitary if $E^{H}(z) E(z)=I$ , where the superscript $H$ stands for the conjugated transpose, and I is the identity matrix [23]. A perfect factorization of the paraunitary matrix with or without constraints often gives an 
effective structure which provides optimal design and fast implementation.

In [32], factorization of paraunitary matrices in the framework of M-channel maximally decimated FIR filter banks is investigated. The results can be applied to M-band wavelets as well as multi wavelet systems. Based on the singular value decomposition of the coefficient matrices, any constrained paraunitary matrix of order k-1 can be expressed as the product of k-1 order-one paraunitary building blocks and an additional unitary matrix. These factorizations provide efficient structures for implementing linear-phase and mirror image paraunitary filter banks with length constraint. More recently, Rault and Guillemot proved that an $\mathrm{M} \times \mathrm{M}$ paraunitary matrix of order K-1 can be factorized into K-1 stage order-one form if the ranks of the first and last coefficient matrices are not less than $M / 2$ for even or $(M-1) / 2$ for odd M.

However its structure is still redundant, thus a simpler structure is developed in [33] which Presents a novel lattice structure of the generalized M-channel paraunitary filter banks. By removing the redundancy of the lattice structure, the minimal and complete structure for which the number of multiplication and addition is less than the conventional method is derived.

Though PUFBs present good coding results for Lossy image coding, they are not applied to lossless one. In [35], by reducing the redundant parameters in Householder matrix, a novel lifting structure for PUFBs which has less implementation costs similar to [34] are not only applied to lossless image coding but also lossy image coding.

The Polyphase matrix E(z) of the PUFB is represented as

$$
E(z)=X_{L} \Lambda_{L}(z) \cdots X_{1}(z) \Lambda_{1}(z) X_{0}
$$

$$
\text { Where, } \Lambda_{k}(z)=\left[\begin{array}{cc}
I_{\left(M-\gamma_{k}\right)} & 0 \\
0 & z^{-1} I_{\gamma_{k}}
\end{array}\right]
$$

And $X_{k} \mathrm{~s}$ are $\mathrm{M} \times \mathrm{M}$ arbitrary orthogonal matrices. Although $\gamma_{k}$ is arbitrary integer $1 \leq \gamma_{k} \leq M$.For $M \times M$ orthogonal matrix $\mathrm{X}$ Givens Rotation Factorization results into a product of $M(M-1) / 2$ rotation angles $\Theta_{i, j}$ [24]

$$
X=\prod_{i=1}^{M-1} \prod_{j=i+1}^{M} \Theta_{i, j}
$$

$$
\left[\Theta_{i, j}\right]=\left\{\begin{array}{cc}
1 & : k=l \neq i \text { or } j \\
\cos \theta_{i, j} & : k=l=i \text { or } j \\
-\sin \theta_{i, j} & : k=i \text { and } l=j \\
0 & \text { :otherwise }
\end{array}\right.
$$

A lattice structure presented in [34] has less implementation cost than that in [33]. The lattice structure in [34] is represented as

$$
E(z)=\left(\prod_{k=L}^{1} \tilde{X}_{k} \Lambda(z)\right) X_{0}
$$

Where,

$$
\tilde{X}_{k}=\prod_{i=1}^{M / 2} \prod_{j=M / 2+1}^{M} \Theta_{i, j}
$$

The above equation corresponds to separate $M / 2$ paths with delay and $M / 2$ paths without delay and construct the Givens rotation matrix from each path with delay to each path without delay. Also the matrix $X_{0}$ is an arbitrary $M \times M$ orthogonal matrix and includes $M(M-1) / 2$ free parameters. On the other hand, the matrix $X_{k}$ except for $X_{0}$ includes $(M / 2)^{2}$ free parameters. Therefore the number of free parameters of [34] is $(K-1) M^{2} / 4+M(M-1) / 2$ and the same as [33]. However the number of addition and multiplication is less than [33].

In [35], A Householder matrix is represented as

$$
\mathbf{H}[\mathbf{p}]=\mathbf{I}-2 \mathbf{p p}^{\dagger} \text { where }\|\mathbf{p}\|=1
$$

Where $p=\left[\begin{array}{llll}p_{0} & p_{1} & \cdots & p_{M-1}\end{array}\right]^{T}$ and $(H[p])^{-1}=H[p]$. Any orthogonal matrices can be always factorized into cascading Householder matrices. In addition, $H\left[p_{0}\right]$ which satisfies following equation is exists

$$
H\left[p_{0}\right] X^{(M)}=\left[\begin{array}{cc}
I^{(1)} & 0 \\
0 & X^{(M-1)}
\end{array}\right]
$$

Where $X^{(M-1)}$ is $(M-1) \times(M-1)$ orthogonal matrix . By calculating recursively like (16), author derived the relationship

$$
H\left[p_{M-2}\right] \cdots H\left[p_{1}\right] H\left[p_{0}\right] X(M)=I
$$

Hence, $X(M)$ can be factorized into

$$
X(M)=H\left[p_{0}\right] H\left[p_{1}\right] \cdots H\left[p_{M-2}\right]
$$

A relationship between Givens rotation and Householder matrix factorization is obtained, redundant Givens rotation can be removed, and thus simpler Householder matrices can be calculated. For M-channel case, generalized structure is 


$$
\left[\begin{array}{ccc}
\mid & & \mid \\
p_{0} & \cdots & p_{M / 2-1} \\
\mid & & \mid
\end{array}\right]=\left[\begin{array}{cccc}
\times & 0 & \cdots & 0 \\
0 & \times & \ddots & \vdots \\
\vdots & \ddots & \ddots & 0 \\
0 & \cdots & 0 & \times \\
\times & \times & \cdots & \times \\
\vdots & \vdots & \ddots & \vdots \\
\times & \times & \cdots & \times
\end{array}\right]
$$

Since the number of free parameters is same as [33] a Householder matrix is also factorized into a lifting structure as

$$
H[p]=\left[\begin{array}{ccccccc}
1 & & & & & & \\
& \ddots & & & & & \\
& & 1 & & & & \\
\alpha_{1} & \cdots & \alpha_{r-1} & 1 & \alpha_{r-1} & \cdots & \alpha_{M} \\
& & & & 1 & & \\
& & & & & \ddots & \\
& & & & & & 1
\end{array}\right]
$$

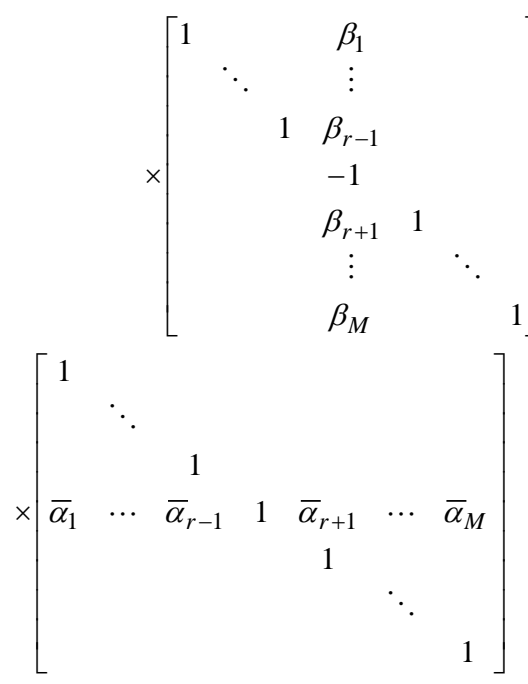

Where $\alpha_{k}, \beta_{k}$ and $\bar{\alpha}_{k}$ are $\alpha_{k}=p_{k} / p_{r}, \beta_{k}=-2 P_{k} P_{r}$ and $\bar{\alpha}_{k}=-\alpha_{k}$

The Householder factorization is a minimal structure of an orthogonal matrix, since the position of the Householder matrices and merge rounding operators is changed. It is obvious that Lifting Based PUFBs (LPPUFBs) based on Householder matrices always have less number of rounding operators than Givens rotation matrix ones.

It is shown that, method proposed in [35] gives better results on the PSNR against 9/7-tap WT and the high frequency region of the reconstructed image using the LBPUFB is well approximated. LBPUFBs presented in [35] shows superior coding results on the entropy and the PSNR against 9/7- and 5/3-tap Wavelets to both Lossy/lossless images coding, respectively.

\subsection{Bi-orthogonal Filter Banks (BOFBs)}

Although PUFBs can be designed easily and also the number of design parameters is smaller than that of BOFBs, the frequency responses of PUFBs are usually worse. Also PUFB's have many restrictions compared to BOFBs.To apply FBs to lossless image coding, the lattice structure should be represented by lifting structures which has unity diagonal scaling coefficients to avoid quantization errors. Factorization of BOFBs involves non-unity diagonal scaling coefficients. Hence, they have not been applied to lossless coding directly. In [36], degree-1 BOFBs which have unity diagonal scaling coefficients throughout the lifting structure have been proposed. In [37], order-1 building blocks in BOFBs are took forward. Though this lifting structure has more design parameters than those of the conventional order-1 PUFBs

And degree-1 BOFBs, structure shown in [37] provide reduction in the number of rounding operations since a rounding operation can be regarded as quantization noise; the number of rounding operations affects the subband energy compaction. Therefore, the number of rounding operations should be minimized as much as possible.

The structure shown in [37] has taken into account not only restriction such as paraunitary but also has rounding operation less compared to [30] and [40].

But still the structure in [37] did not consider the restrictions such as number of channels and McMillan degree. The blocklifting structure proposed in [38] covers broader family which gives best solution compared to the conventional methods. This structure is free from the restriction such as Paraunitary, number of channels and McMillan degree while maintaining less rounding operations than [39] and [40]. Compared to conventional FBs, the designed BOFBs give better Lossy-tolossless image coding performance in both PSNRs and perceptual visual quality for the images containing a lot of high frequency components [38].

\section{DISCUSSION AND CONCLUSION}

In many applications, image coding is playing vital role in every aspect. This review presents that advances in lossy coding is growing faster. Still ongoing research has been made this as a center of attraction. Authors tried to figure out a comprehensive and recent progress in this review.

While dealing with lossless compression one cannot enjoy the benefit of high compression rate and when one going for lossy image coding they need to pay for high compression rate at the cost of perceptual quality of an image. The research achievement with the advancement as to utilize filter bank structure as a lifting scheme with integer-to-integer transform, advantage of reduced number of rounding operation and design structure that is free from restriction is enjoyed, but certainly even superior structure are needed.

Here, superior means a structure that has negligible number of rounding operations. A number of rounding operations shows some amount of quantization error which degrades the image quality. The filter coefficient or factorized coefficient which nearly approximates to inter value can prove to be efficient in terms of rounding operations that are performed while lifting.

Similarly, the filter bank structure needs to show best performance for the image which contains a lot of low frequency regions. At the same time, "superior" also means faster computation algorithm with the simpler structure.

Since the Demand from the multimedia user is growing at a faster rate the algorithm which overtook that demand finding a huge need. 
Table 1 Coding Gain in DB of 8-channel Filter Banks

\begin{tabular}{|c|c|c|c|c|}
\hline Method & $\begin{array}{c}\text { DCT }(8 \\
\text { x 8) }\end{array}$ & $\begin{array}{c}\text { LOT(8 x } \\
16)[10]\end{array}$ & $\begin{array}{c}\text { PUFB }(8 \\
\text { X 16) } \\
{[38]}\end{array}$ & $\begin{array}{c}\text { BOFB }(8 \\
\text { x 16 }) \\
{[38]}\end{array}$ \\
\hline Coding gain & 8.83 & 9.22 & 9.35 & 9.62 \\
\hline
\end{tabular}

Table 2 PSNR Results in DB for Barbara using Various Transforms.

\begin{tabular}{|c|c|c|c|}
\hline Method & 0.25 bpp & 0.5 bpp & 1.0 bpp \\
\hline DCT( 8 x 8)[18] & 27.28 & 31.08 & 36.25 \\
\hline $\begin{array}{c}\text { LOT (8 x 16) [10] } \\
\text { GenLOT (8 x24) } \\
{[18]}\end{array}$ & 33.91 & 36.13 & 39.28 \\
\hline $\begin{array}{c}\text { DWT 9/7-tap [38] } \\
\text { PUFB (8 x 16) [38] }\end{array}$ & 33.71 & 36.83 & 40.03 \\
\hline BOFB (8 x 16) & 32.98 & 36.33 & 39.82 \\
\hline
\end{tabular}

\section{REFERENCES}

[1] Bahl L. R., Kobayashi H, "Image Data Compression by Predictive Coding I: Prediction Algorithms", IBM Journal of Research \& Development, vol. 18, no. 2, 1974.

[2] Wen-Jun Zhang Song-Yu Yu Hong-Bin Chen, "A new adaptive classified transform coding method [image coding]" International Conference on Acoustics, Speech \& Signal Processing, 1989.

[3] M. Antonini, M. Barlaud, P. Mathieu, and I. Daubchies, "Image coding using wavelet transform," IEEE Trans. Image Process., vol. 1, no. 4, pp. 205-220, Apr. 1992.

[4] Budge S., Baker R, "Compression of color digital images using vector quantization in product codes", IEEE Trans. Image Process., vol. 10, no. 4, pp. 129-132, Apr. 1985.

[5] Anderson G., Huang T., "Piecewise Fourier Transformation for Picture Bandwidth Compression", IEEE Trans. Communication Technology, vol. 19, no. 2, pp. 133-140, Apr.1971

[6] Ready P, Wintz P, "Information Extraction, SNR Improvement, and Data Compression in Multispectral Imagery", IEEE Trans. Communications, vol. 21, no. 10, pp. 1123-1131, Oct. 1973.
[7] Feria, Erlan H. Barba, Joseph Scheinberg, Norman, "A Simple Predictive Transform Coder for Images" IEEE Military Communications Conference - Communications - Computer, 1986.

[8] Andrews H., Patterson C., "Singular Value Decomposition (SVD) Image Coding ", IEEE Trans. Communications, vol. 24, no. 4, pp. 425-432, Apr. 1976.

[9] Michael D.A.(2002), "Reversible integer-to-integer transforms for image coding",(Phd thesis),University of British Columbia

[10] H. S. Malvar and D. H. Staelin, "The LOT: Transform coding without blocking effects," IEEE Trans. Acoustic., Speech, Signal Process., vol. 37,no. 4, pp. 553-559, Apr. 1989.

[11] H. C. Reeve. III. And J. S. Lim."Reduction of blocking effect in image coding." in Proc. 1CASSP 83. Boston. MA. pp. 1212-1215., Apr.1983.

[12] D. E. Pearson and M. W. Whybray, "Transform coding of images using interleaved blocks," IEEE Proc., Part F, vol. 131, .pp. 466-472, Aug. 1984.

[13] H. S. Malvar, "A pre- and post-filtering technique for the reduction of blocking effects," presented at the Picture Coding Symp., Stock- holm, Sweden, June 1987.

[14] N. Ahmed and K. R. Rao," Orthogonal transforms for digital signal processing." New York, NY: Springer, 1975.

[15] H. S. Malvar, "Signal Processing with Lapped Transforms". Norwood, MA: Artech House, 1992.

[16] P. Cassereau, "A New Class of Optimal Unitary Transforms for Image Processing", Master's Thesis, Mass. Inst. Tech., Cambridge, MA, May 1985.

[17] K. R. Rao and P. Yip "Discrete Cosine Transform: Algorithms, Advantages, Applications", San Diego, CA: Academic Press, 1990.

[18] R. L. de Queiroz, T. Q. Nguyen, and K. R. Rao, “The GenLOT: Generalized linear-phase lapped orthogonal transform," IEEE Trans. Signal Process., vol. 44, no. 3, pp. 497-507, Mar. 1996.

[19] C. K. Chui (ed.), "Wavelets - A Tutorial in Theory and Applications", San Diego, CA: Academic Press, 1992.

[20] A. N. Akansu and R. A. Haddad," Multiresolution Signal Decomposition: Transforms, Subband, Wavelets", San Diego, CA: Academic Press, 1992.

[21] W. Sweldens "The lifting scheme: A construction of second generation wavelets," SIAM J. Math. Anal., vol. 29, no. 2, pp. 511-546, 1997.

[22] W. Sweldens, "The lifting scheme: A new philosophy in biorthogonal wavelet constructions," in Proc. of SPIE $2569,1995$.

[23] R. E. Crochiere and L. R. Rabiner, Multirate Digital Signal Processing. Englewood Clis, NJ: Prentice-Hall, 1983.

[24] P. P. Vaidyanathan, Multirate Systems and Filter Banks. Englewood Cliffs, NJ: Prentice Hall, 1992.

[25] D. Esteban and C. Galand, "Applications of quadrature mirror to split band voice coding schemes". Acoustics, Speech, and Signal Processing, IEEE International Conference on ICASSP '77.vol.2,pp.191-195,May 1977.

[26] J. D. Johnston, A filter family designed for use in quadrature mirror filter banks, "Proc. of Intl. Conf. on 
Acoust. Speech, Signal Processing, Denver, CO, pp. $291\{294,1980\}$.

[27] M. J. Smith and T. P. Barnwell, "Exact reconstruction techniques for tree structured Subband coders", IEEE Trans. Acoust., vol.34,no.3,pp.434-441,Jun 1986.

[28] M. Vetterli and D. Le Gall, "Perfect reconstruction filter banks: some properties and factorizations", IEEE Trans. Acoust., Speech, Signal Processing, ASSP-37, pp.1057 \{1071, July 1989\}.

[29] A. V. Oppenheim and R. W. Schafer, Digital Signal Processing, Englewood's Cliffs,NJ : Prentice-Hall, 1975.

[30] D. Le Gall, and A. Tabatabai "Sub-band coding of digital images using symmetric short kernel filters and arithmetic coding techniques" Proc. of Intl. Conf. onAcoust., Speech, Signal Processing, pp. 761\{764, $1988\}$.

[31] R.L. de Queiroz and K. R. Rao, "Time-varying lapped transforms and wavelet packets", IEEE Trans. on Signal Processing, vol. 41, pp. 3293\{3305, Dec. 1993\}.

[32] R. L. de Queiroz and H. S. Malvar, "On the asymptotic performance of hierarchical transforms", IEEE Trans. on Signal Processing, Vol 40, pp. 2620 \{2622, Oct. 1992\}.

[33] X. Gao, T. Q. Nguyen, and G. Strang, "On factorization of M channel paraunitary filter banks," IEEE Trans. Signal Process. Vol. 49, no. 7, pp. 1433-1446, Jul. 2001.
[34] M. Ikehara and Y. Kobayashi, "A novel lattice structure of M channel paraunitary filter banks," in Proc., IEEE Int. Symp. Circuits Syst., pp. 4293-4296, 2005.

[35] T. Suzuki, Y. Tanaka, and M. Ikehara, "Lifting-based paraunitary filter banks for Lossy/lossless image coding," IEICE Trans. Fundamentals,vol. J89-A, no. 11, pp. 950-959, Nov. 2006.

[36] Y.-J. Chen, S.Oraintara, and K. S. Amaratunga, "Mchannel lifting-based design of paraunitary and biorthogonal filter banks with structural regularity," in Proceedings of ISCAS '03., Bangkok, Thailand, May 2003.

[37] S. Iwamura, Y. Tanaka, and M. Ikehara, "An efficient lifting structure of biorthogonal filter banks for lossless image coding," in Proc. of ICIP'07, San Antonio, TX, Sep. 2007, pp. 433-436.

[38] Taizo Suzuki , Masaaki Ikehara, and Truong Q. Nguyen," Generalized Block-Lifting Factorization of MChannel Biorthogonal Filter Banks for Lossy-to-Lossless Image Coding" IEEE Transactions on Image process.,vol.21,no.7,pp3220-3228, July2012

[39] P. Hao and Q. Shi, "Matrix factorizations for reversible integer mapping,'IEEE Trans. Signal Process., vol. 49, no. 10, pp. 2314-2324, Oct. 2001

[40] Y. She, P. Hao, and Y. Paker, "Matrix factorizations for parallel integer transformation," IEEE Trans. Signal Process. vol. 54, no. 12, pp. 4675-4684, Dec. 2006. 